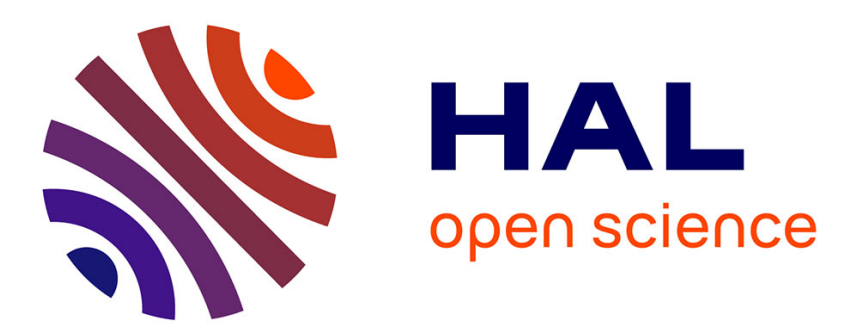

\title{
Spatiotemporal optical solitons in carbon nanotube arrays
}

\author{
Hervé Leblond, Dumitru Mihalache
}

\section{To cite this version:}

Hervé Leblond, Dumitru Mihalache. Spatiotemporal optical solitons in carbon nanotube arrays. Physical Review A: Atomic, molecular, and optical physics [1990-2015], 2012, 86 (4), pp.043832. 10.1103/PhysRevA.86.043832 . hal-03187678

\section{HAL Id: hal-03187678 \\ https://univ-angers.hal.science/hal-03187678}

Submitted on 1 Apr 2021

HAL is a multi-disciplinary open access archive for the deposit and dissemination of scientific research documents, whether they are published or not. The documents may come from teaching and research institutions in France or abroad, or from public or private research centers.
L'archive ouverte pluridisciplinaire HAL, est destinée au dépôt et à la diffusion de documents scientifiques de niveau recherche, publiés ou non, émanant des établissements d'enseignement et de recherche français ou étrangers, des laboratoires publics ou privés. 


\title{
Spatiotemporal optical solitons in carbon nanotube arrays
}

\author{
Hervé Leblond ${ }^{1}$ and Dumitru Mihalache ${ }^{1,2,3}$ \\ ${ }^{1}$ LUNAM Université, Université d'Angers, Laboratoire de Photonique d'Angers, EA 4464, 2 Boulevard Lavoisier, \\ 49045 Angers Cedex 01, France \\ ${ }^{2}$ Academy of Romanian Scientists, 54 Splaiul Independentei, Bucharest 050094, Romania \\ ${ }^{3}$ Horia Hulubei National Institute for Physics and Nuclear Engineering, Magurele-Bucharest 077125, Romania
}

(Received 13 June 2012; published 23 October 2012)

\begin{abstract}
We consider the formation of ultrashort spatiotemporal optical waveforms in arrays of carbon nanotubes. We use a short-wave approximation to derive a generic two-dimensional sine-Gordon equation, describing ultrashort soliton evolution in such nanomaterials. This model was derived by using a rigorous application of the reductive perturbation formalism (multiscale analysis) for the Maxwell equations and for the corresponding Boltzmann kinetic equation for the distribution function of electrons in carbon nanotubes. We show numerically diffractionless and dispersionless robust propagation over large distances (with respect to the wavelength) of few-cycle $(2+1)$-dimensional spatiotemporal solitons in the form of optical breathers.
\end{abstract}

DOI: 10.1103/PhysRevA.86.043832

PACS number(s): 42.65.Tg, 42.65.Sf, 47.20.Ky, 73.63.Fg

\section{INTRODUCTION}

Carbon nanotubes (CNTs) are believed to be the wonder material of this century due to the tremendous range of physical, mechanical, thermal, electronic, and optical properties offered by such nanomaterials [1-3]. The unique characteristics of CNTs have generated a strong interest in their possible use in nanomechanical, nanoelectronic, and nanophotonic devices. Emerging applications of CNTs using their good thermal and electronic conductivities are flat panel displays, field-effect transistors, etc. The use of these nanomaterials in biosensors, chemical and pressure sensors, as well as in biomedical applications, e.g., as drug delivery vehicles, are also being envisaged. CNTs were synthesized in 1991 as graphitic carbon needles [4], ranging from 4 to $30 \mathrm{~nm}$ in diameter and up to $1 \mu \mathrm{m}$ in length. That pioneering paper of Iijima [4] was of particular importance because it brought CNTs to the awareness of the scientific community as a whole. Then, the large-scale synthesis [5] of carbon nanometer-size tubes provided an impetus to research in the area of carbon fiber growth as well as in the production and characterization of fullerene-related materials. The synthesis of abundant single-shell tubes with diameters of about $1 \mathrm{~nm}$ was reported as early as 1993 [6]. Note that, whereas, the multishell CNTs are formed on the carbon cathode, the single-shell tubes grow in the gas phase, see Ref. [6].

Comprehensive studies of various nonlinear effects in CNT arrays were performed in the past few years. Here, we mention that discrete breathers, that is, intrinsic localized modes or discrete solitons [7], which appear in strongly nonlinear systems being responsible for energy localization in discrete anharmonic lattices were studied in CNT arrays [8]. Largeamplitude oscillating modes of carbon nanotubes that have the additional features of being nonlinear as well as discrete were considered in Ref. [8]. Nonlinear spin-wave propagation in CNTs has also been considered [9]. A study of propagation of short optical pulses in CNTs placed in dispersive nonmagnetic dielectric media was reported recently [10]. We also mention the recent paper on the propagation of extremely short optical pulses in coupled graphene waveguides [11]; these $(1+1)$ dimensional solitons can be considered as discrete optical solitons [12], which form in such graphene waveguides.
In Ref. [13], we used a powerful reductive perturbation technique or a multiscale analysis [14] to obtain a generic Kadomtsev-Petviashvili evolution equation governing the propagation of $(2+1)$-dimensional femtosecond spatiotemporal optical solitons, alias light bullets (LBs) $[15,16]$ in quadratic nonlinear media beyond the slowly varying envelope approximation (SVEA). The collapse of ultrashort spatiotemporal pulses in cubic (Kerr-like) media was also studied by using the multiscale analysis beyond the SVEA, and it was obtained from the Maxwell-Bloch equations for two-level atoms a generic cubic generalized Kadomtsev-Petviashvili nonlinear evolution equation [17]. Ultrashort LBs described by the two-dimensional sine-Gordon (2DsG) equation, obtained by using the multiscale analysis in the short-wave approximation for a cubic (Kerr-like) nonlinear medium, were studied recently [18], and it was shown that robust few-cycle LBs may form, oscillating in both space and time, that is, two-dimensional breathers.

In this paper, we derive, in a rigorous way by using the reductive perturbation method [14] in the short-wave approximation regime, the governing model in the form of a $2 \mathrm{DsG}$ equation for the propagation of $(2+1)$-dimensional spatiotemporal optical solitons in arrays of carbon nanotubes. We show that a perturbed few-cycle plane-wave input evolves into a robust two-dimensional $\mathrm{LB}$, which propagates without being dispersed and diffracted over a few millimeters (thus, the propagation distance is very long with respect to the wavelength, ensuring the validity of the short-wave approximation).

\section{GOVERNING MODEL FOR LIGHT PROPAGATION IN CNT ARRAYS}

Belonenko and co-workers recently derived a model which accounts for light propagation across an array of carbon nanotubes [19,20]. They numerically solved their model equation and found evidence for soliton propagation. In two dimensions, they claimed that the numerical results show $(2+1)$-dimensional light-bullet propagation [20] in arrays of CNTs, but the two-dimensional spatiotemporal waveform they display is not a genuine spatiotemporal optical soliton because it is spread out due to diffraction. We will see that the model, 


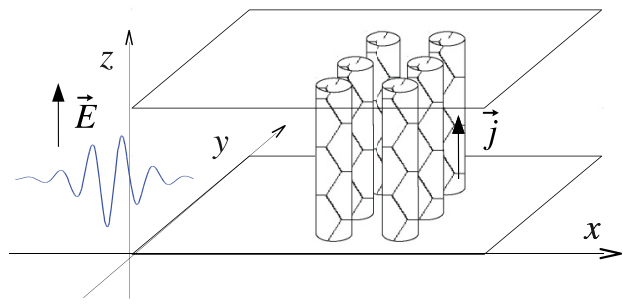

FIG. 1. (Color online) Geometry of the problem.

derived in Refs. [19,20], can be reduced with a slight further approximation to the sine-Gordon equation. In two dimensions, the so-called two-dimensional sine-Gordon equation is obtained. It admits light-bullet solutions, which are oscillating ones, i.e., breatherlike spatiotemporal optical solitons.

In the absence of charge density and of magnetization, using the gauge $\vec{E}=-\partial \vec{A} / \partial t$, the Maxwell equations reduce to the equation,

$$
\Delta \vec{A}-\frac{1}{c^{2}} \frac{\partial^{2} \vec{A}}{\partial t^{2}}=-\mu_{0} \vec{j}
$$

to be satisfied by the vector potential $\vec{A}$. Here, $\mu_{0}$ and $c$ are the magnetic permeability and the light velocity in vacuum, respectively, and we use Système International units. In the considered geometry (see Fig. 1), the current $\vec{j}$ is directed along the direction of the nanotubes ( $z$ axis) $\vec{j}=j \vec{e}_{z}$, where $\vec{e}_{z}$ is a unitary vector directed along $z$. We assume that the wave field is polarized in the same direction, and $\vec{A}=A \vec{e}_{z}$. Here, $A$ and $j$ are functions of $x, y$, and $t$. The Laplacian operator in Eq. (1) reduces, thus, to $\Delta=\partial^{2} / \partial x^{2}+\partial^{2} / \partial y^{2}$. The current is related to the vector potential by means of a semiclassical approach, which uses the classical Boltzmann kinetic equation,

$$
\frac{\partial f}{\partial t}-q \frac{\partial A}{\partial t} \frac{\partial f}{\partial p}=\frac{F_{0}-f}{\tau},
$$

in which $q$ is the electron charge, $f=f(\vec{p}, t)$ is the distribution function of electrons in the nanotubes in which $\vec{p}=\left(p_{\varphi}, p_{z}\right)$ is the momentum of the electron, where $p_{\varphi}=s \Delta p_{\varphi}$ is the azimuthal component, and the axial component $p_{z}$ is merely denoted by $p$ below. Hence, the integer $s$ characterizes the momentum quantization transverse to the nanotube. The equilibrium value $F_{0}$ of the distribution function $f$ is the Fermi-distribution function,

$$
F_{0}=\frac{1}{1+\exp \left(E / k_{B} T_{0}\right)},
$$

in which $k_{B}$ is the Boltzmann constant, $T_{0}$ is the absolute temperature, and the energy $E$ in the conduction band in the case of zigzag CNTs [21] is given by

$$
E=\gamma \sqrt{1+4 \cos (a p) \cos \frac{\pi s}{m}+4 \cos ^{2} \frac{\pi s}{m}} .
$$

Here, we have $\gamma=2.7 \mathrm{eV}, a=3 b / 2 \hbar$, where $b=0.142 \mathrm{~nm}$ is the distance between adjacent carbon atoms and $m$ is the number of hexagons in the perimeter of a nanotube. Then, the surface current density $j_{s}$ is given by

$$
j_{s}=\frac{2 q}{(2 \pi \hbar)^{2}} \iint v f d p_{\varphi} d p
$$

where the velocity $v$ is $v=\partial E / \partial p$. The distribution function $f$ can be written as

$$
f=\sum_{s} \Delta p_{\varphi} \delta\left(p_{\varphi}-s \Delta p_{\varphi}\right) f_{s}(p, t) .
$$

Here, $f_{s}$ is the longitudinal distribution function relative to the azimuthal quantum number $s$. The integration over $p_{\varphi}$ is straightforward, and the separation between two values of $p_{\varphi}$ is $\Delta p_{\varphi}=2 \pi \hbar / l$, where $l=n b \sqrt{3}$ is the perimeter of the nanotube.

Denoting the surface density of nanotubes in the $x y$ plane by $N$, the volume current density $j$ is deduced from the surface one (5) as

$$
j=\frac{N q}{\pi \hbar} \sum_{s} \int v f_{s} d p .
$$

Note that the distinction between the two currents $j_{s}$ and $j$ and, consequently, the dependence with respect to $N$ in Eq. (7) were missed in Refs. [19,20].

Instead of the relatively complicated derivation of the governing equations for light-bullet propagation in CNT arrays, which was performed in Ref. [19], we use the powerful reductive perturbation method in the short-wave approximation regime [14,22]. We assume that the typical duration of the pulse (in the optical range) is very small with respect to $\tau$ but that the propagation length is very long with respect to the wavelength. We, thus, introduce fast and slow variables,

$$
\theta=\frac{1}{\varepsilon}\left(t-\frac{x}{V}\right), \quad \xi=\varepsilon x,
$$

in which $\varepsilon$ is a small parameter and $V$ is a wave velocity to be determined. The fast variable $\theta$ accounts for the shape of the wave in the frame moving at velocity $V$, whereas, the slow variable $\xi$ holds for the propagation distance. The dependent variables are expanded in a power series of $\varepsilon$ as $f_{s}=f_{0}+$ $\varepsilon f_{1}+\cdots$, and so on. The derivation operators,

$$
\frac{\partial}{\partial t}=\frac{1}{\varepsilon} \frac{\partial}{\partial \theta}, \text { and } \frac{\partial}{\partial x}=\varepsilon \frac{\partial}{\partial \xi}-\frac{\varepsilon}{V} \frac{\partial}{\partial \theta}
$$

are then reported in Eqs. (1) and (2). At leading order $1 / \varepsilon$, Eq. (2) yields

$$
\frac{\partial f_{0}}{\partial \theta}-q \frac{\partial A_{0}}{\partial \theta} \frac{\partial f_{0}}{\partial p}=0 .
$$

The general solution of Eq. (9) is $f_{0}=\varphi\left(p+q A_{0}\right)$, where $\varphi$ is some arbitrary function. At large $t$, the wave $A$ vanishes, and $f_{0}$ goes to its equilibrium value $F_{0}$, hence, $\varphi=F_{0}$. Reporting this expression of $f$ in Eq. (7) and making the change in variable $p \rightarrow p-q A$, we get

$$
j_{0}=\frac{q}{\pi \hbar} \sum_{s} \int v(p-q A) f_{0}(p) d p,
$$

which yields the same expression as used in Ref. [19].

Reporting the fast and slow variables in the Maxwell equation (1) yields, at leading order $1 / \varepsilon^{2}$,

$$
\frac{1}{V^{2}} \frac{\partial^{2} A_{0}}{\partial \theta^{2}}-\frac{1}{c^{2}} \frac{\partial^{2} A_{0}}{\partial \theta^{2}}=0,
$$


and consequently, $V=c$. The equation at order $1 / \varepsilon$ is automatically satisfied, and we get, at order $\varepsilon^{0}$,

$$
\frac{-2}{c} \frac{\partial^{2} A_{0}}{\partial \xi \partial \theta}=-\mu_{0} j_{0} .
$$

Equations (10) and (12) yield the governing model.

\section{REDUCTION TO THE SINE-GORDON EQUATION}

Then, instead of expanding $v(p)$ in a Fourier series as in Ref. [19], let us consider the Boltzmann distribution $F_{0}$. The energy $E$ is on the same order of magnitude as $\gamma \simeq 2.7 \mathrm{eV}$, hence, $\gamma / k_{B} \simeq 3.1 \times 10^{4} \mathrm{~K}$, which is very large with respect to room temperature. This means that only the levels with the lowest energy are excited. Let us now seek this minimum. For a given $s$, elementary analysis shows that the minimum value of $E$ is

$$
E_{m}=\gamma|1-2| \cos \frac{\pi s}{m}||,
$$

and is reached for $p= \pm \pi / a$ if $\cos \pi s / m>0$ and is reached for $p=0$ if $\cos \pi s / m<0$. Assuming, for the analysis, that $s$ can take any real value, the minimum of $E_{m}$ as $s$ varies is 0 and is obtained if $\cos \pi s / m= \pm 1 / 2$, i.e., for $s / m= \pm 1 / 3$ or $\pm 2 / 3$. If $m=6$, e.g., the minimum will be reached for $s=2$ and 4 only. Since $s$ is an integer, the value $E=0$ is reached for values of $m$, which are multiples of 3 only. In the other nanotubes, there is a nonzero gap between valence and conduction bands. Then, according to the value of $\gamma$, the conductivity will be very low. Hence, only the nanotubes with $m$ multiples of 3 will contribute. The expression reduces to

$$
E_{s}(p)=2 \gamma\left|\cos \frac{a p}{2}\right|
$$

for $s / m=1 / 3$ and to

$$
E_{s}(p)=2 \gamma\left|\sin \frac{a p}{2}\right|
$$

for $s / m=2 / 3$. Taking the derivative of Eq. (14) [respectively, (15)] with respect to $p$, then substituting $p-q A_{0}$ for $p$ and considering the value at which the minimum is reached, $p=$ $\pi / a$ (respectively, $p=0$ ) yields

$$
v=-a \gamma \operatorname{sgn}\left(\sin \frac{a q A_{0}}{2}\right) \cos \frac{a q A_{0}}{2},
$$

where $\operatorname{sgn}(X)$ denotes the sign of $X$ in both cases. Since the essential contribution to the integral in Eq. (10) comes from the minimum of $E$, we can replace $v$ with its expression at the minimum (16), in the integral (10). The integrals,

$$
I_{s}=\int_{-\pi / a}^{\pi / a} \frac{d p}{1+\exp \left[E_{s}(p) / k_{B} T\right]}
$$

can be reduced to

$$
I_{m / 3}=I_{2 m / 3}=\frac{2}{a} \Phi\left(\frac{\gamma}{k_{B} T}\right),
$$

with

$$
\Phi(X)=\int_{-\pi / 2}^{\pi / 2} \frac{d x}{1+e^{2 X|\sin x|}} .
$$

Finally, we get the following expression for the current:

$$
j_{0}=-Q \operatorname{sgn}\left(\sin \frac{a q A_{0}}{2}\right) \cos \frac{a q A_{0}}{2},
$$

in which the constant $Q$ is

$$
Q=\frac{4 N q \gamma}{\pi \hbar} \Phi\left(\frac{\gamma}{k_{B} T_{0}}\right) .
$$

Inserting Eq. (20) into Eq. (12) yields the evolution equation. However, due to the sign of the sine term, it presents a discontinuity as $A_{0}=0$. It is clear that such a discontinuity is unphysical. Recall, however, that $A_{0}$ is a component of a potential and, hence, is only defined up to an additive constant. Let us assume $0<a q A_{0} / 2<\pi$ and define $A_{0}^{\prime}$ such that $a q A_{0}^{\prime} / 2=a q A_{0} / 2-\pi / 2$. Then, Eq. (12) reduces to

$$
\frac{\partial^{2} A_{0}^{\prime}}{\partial \xi \partial \theta}=-R \sin \frac{a q A_{0}^{\prime}}{2},
$$

which is exactly the sine-Gordon equation, and where we have set

$$
R=\frac{2 N q \gamma}{\pi \varepsilon_{0} \hbar c} \Phi\left(\frac{\gamma}{k_{B} T_{0}}\right) .
$$

If $\left|a q A_{0} / 2\right|>\pi$, we can set $A_{0}^{\prime \prime}=A_{0}+\pi / a q$, and we see that the same equation as (22) is satisfied by $A_{0}^{\prime \prime}$. Since $A_{0}, A_{0}^{\prime}$, and $A_{0}^{\prime \prime}$ represent exactly the same electric field and the evolution law of the latter cannot present a discontinuity, this proves that Eq. (22) is, in fact, valid for any $A_{0}$.

\section{LIGHT BULLETS}

If we retain the second transverse derivative in the wave equation (12), the final equation is

$$
\frac{\partial^{2} A_{0}^{\prime}}{\partial \xi \partial \theta}=\frac{c}{2} \frac{\partial^{2} A_{0}^{\prime}}{\partial y^{2}}-R \sin \frac{a q A_{0}^{\prime}}{2},
$$

which is known as the $2 \mathrm{DsG}$ equation. The $2 \mathrm{DsG}$ equation admits light-bullet solutions.

The 2DsG equation is solved numerically as follows: First, we rewrite it in the dimensionless form,

$$
\frac{\partial B}{\partial Z}=U \sin \left(\int^{T} B\right)+\int^{T} \frac{\partial^{2} B}{\partial Y^{2}},
$$

by means of the change in variables $B=E_{0} / E_{r}, Z=$ $x / L_{r}, T=(t-x / c) / t_{r}, \quad$ and $\quad Y=y / w_{r}$, with $E_{r}=$ $2 /\left(t_{r} a q\right), L_{r}=-U E_{r} / R$, and $w_{r}=\sqrt{c t_{r} L_{r} / 2}$. We used $t_{r}=2.785 \mathrm{fs}$ and $U=-10$ for practical reasons, but formally, $U$ is arbitrary, and it has no physical meaning here, in contrast to the case of the two-level systems [23]. Then, (25) is transformed into the rational differential system,

$$
\begin{gathered}
\frac{\partial A}{\partial T}=-B C, \\
\frac{\partial C}{\partial T}=A B \\
\frac{\partial B}{\partial Z}=C+\int^{T} \frac{\partial^{2} B}{\partial Y^{2}},
\end{gathered}
$$

with the assumption that $A$ tends to the fixed value $U$ as $T$ tends to $-\infty$. System (28) is solved by means of a modified 


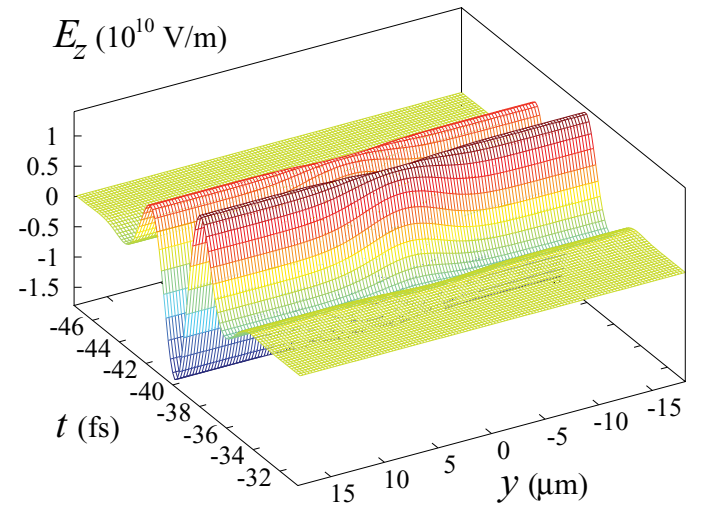

(a)

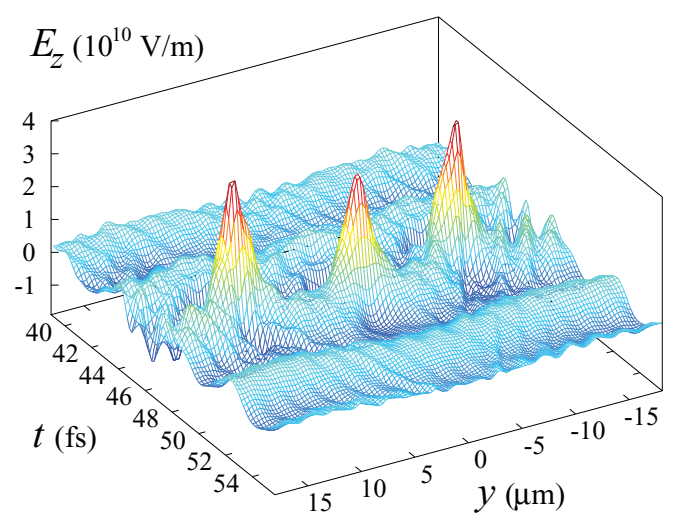

(b)

FIG. 2. (Color online) Evolution of a perturbed plane-wave fewcycle spatiotemporal waveform into two-dimensional light bullets. (a) Input and (b) At $x=720 \mu \mathrm{m}$.

Euler scheme (also known as the midpoint method, a secondorder Runge-Kutta one) in $Z$ in each substep of which the equations relative to the variable $T$ are solved by means of a scheme of the same type. The surface density of nanotubes $N$ is evaluated as follows: We assume that the separation between nanotubes is $d=0.34 \mathrm{~nm}$ and, for the sake of simplicity, that the nanotubes are arranged on a square array and have all the same radii $r=m b \sqrt{3}$ corresponding to $m=6$. Then, $N=(1 / d+2 r)^{2} \simeq 1.5 \times 10^{18} \mathrm{~m}^{-2}$.

First, we consider an initial datum in the form of a perturbed few-cycle plane wave as

$$
E_{z}=\mathcal{A} e^{-\left(t-t_{1}\right)^{2} / w_{t}^{2}} \cos \left[\frac{2 \pi c}{\lambda}\left(t-t_{1}\right)+\varphi\right],
$$

with $t_{1}=\delta t \exp \left(-y^{2} / w_{y}^{2}\right)$ accounting for a transverse perturbation. We used $\lambda=1.25 \mu \mathrm{m}, w_{t}=2.785 \mathrm{fs}, w_{y}=$ $4.83 \mu \mathrm{m}, \varphi=0.233 \pi, \delta t=0.28 \mathrm{fs}, \quad$ and $\mathcal{A}=1.78 \times$ $10^{10} \mathrm{~V} / \mathrm{m}$, which corresponds to the huge peak powers which can be reached in the few-cycle regime. In Fig. 2, it is seen that the initial plane wave breaks into two-dimensional spots. Then, a rough fit of one of these spots is used as the input spatiotemporal waveform to check its stability on propagation as

$$
E_{z}=\mathcal{A} e^{-t^{2} / w_{t}^{2}-y^{2} / w_{y}^{2}} \cos \left[\frac{2 \pi c}{\lambda}\left(t-t_{1}\right)\right]
$$
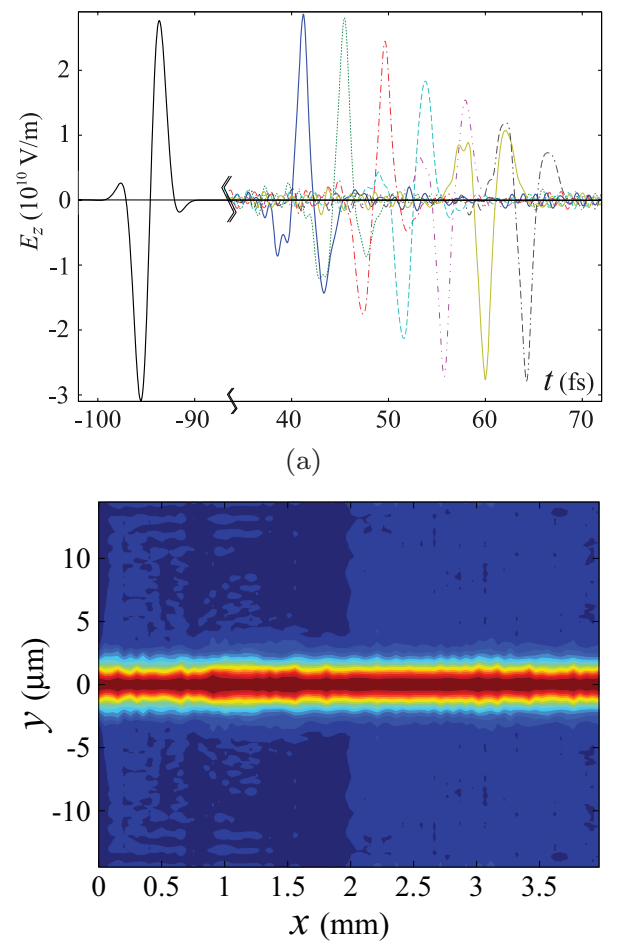

(b)

FIG. 3. (Color online) Evolution of an input-localized few-cycle pulse, which is close to the output two-dimensional light bullet. Panel (a) shows the temporal profile. The solid line on the left shows the input, the lines on the right show the temporal profiles at $x=3.62,3.67,3.72,3.77,3.82,3.87$, and $3.92 \mathrm{~mm}$, showing the oscillations of the two-dimensional breather over a half period. Panel (b) illustrates the diffraction-free evolution (spatial profile). In fact, it shows $\max _{t} t\left(\left|E_{z}\right|\right) / \max _{t, y}\left(\left|E_{z}\right|\right)$ against $y$ and $x$.

The best fit was obtained with $\lambda=1.5 \mu \mathrm{m}, w_{t}=$ $1.95 \mathrm{fs}, w_{y}=1.81 \mu \mathrm{m}$, and $\mathcal{A}=4 \times 10^{10} \mathrm{~V} / \mathrm{m}$. The results are shown in Fig. 3: The propagation occurs freely from diffraction and dispersion up to distances of a few millimeters.

In conclusion, we have shown the possibility for twodimensional optical light-bullet propagation in arrays of CNTs in the two-cycle regime. It must be noticed that damping was neglected in this paper, however, it should be taken into consideration in future papers. On the other hand, from the point of view of mathematical physics, a systematic study on the stability conditions for the two-dimensional breather of the $2 \mathrm{DsG}$ equation remains to be performed. Such a study would allow better specifying the conditions under which two-dimensional light bullets in CNTs could be effectively produced.

\section{ACKNOWLEDGMENTS}

The work of D.M. was supported, in part, by a Senior Chair Grant from the Région Pays de Loire, France. Support from the Romanian Ministry of Education and Research (Project No. PN-II-ID-PCE-2011-3-0083) is also acknowledged. One of the authors (D.M.) would like to thank N. C. Panoiu for helpful discussions. 
[1] R. Saito, G. Dresselhaus, and M. S. Dresselhaus, Physical Properties of Carbon Nanotubes (Imperial College Press, London, 1998).

[2] S. Dresselhaus, G. Dresselhaus, and P. C. Eklund, Science of Fullerenes and Carbon Nanotubes (Academic, New York, 1996).

[3] J.-C. Charlier, X. Blase, and S. Roche, Rev. Mod. Phys. 79, 677 (2007).

[4] S. Iijima, Nature (London) 354, 56 (1991).

[5] T. W. Ebbesen and P. M. Ajayan, Nature (London) 358, 220 (1992).

[6] S. Iijima and T. Ichihashi, Nature (London) 363, 603 (1993).

[7] S. Flach and C. R. Willis, Phys. Rep. 295, 182 (1998); D. K. Campbell, S. Flach, and Y. S. Kivshar, Phys. Today 57(1), 43 (2004).

[8] A. V. Savin and Y. S. Kivshar, Europhys. Lett. 82, 66002 (2008).

[9] H. Leblond and V. Veerakumar, Phys. Rev. B 70, 134413 (2004).

[10] N. N. Yanyushkina, M. B. Belonenko, and N. G. Lebedev, Opt. Spektrosk. 111, 92 (2011) [Opt. Spectrosc. 111, 85 (2011)].

[11] M. V. Belonenko, N. G. Lebedev, and N. N. Yanyushkina, Fiz. Tverd. Tela 54, 162 (2012) [Phys. Solid State 54, 174 (2012)].
[12] F. Lederer, G. I. Stegeman, D. N. Christodoulides, G. Assanto, M. Segev, and Y. Silberberg, Phys. Rep. 463, 1 (2008).

[13] H. Leblond, D. Kremer, and D. Mihalache, Phys. Rev. A 80, 053812 (2009).

[14] H. Leblond, J. Phys. B 41, 043001 (2008).

[15] Y. Silberberg, Opt. Lett. 15, 1282 (1990).

[16] B. A. Malomed, D. Mihalache, F. Wise, and L. Torner, J. Opt. B 7, R53 (2005); D. Mihalache, Rom. J. Phys. 57, 352 (2012); Proc. Romanian Acad. A 11, 142 (2010); Rom. Rep. Phys. 63, 9 (2011).

[17] H. Leblond, D. Kremer, and D. Mihalache, Phys. Rev. A 81, 033824 (2010)

[18] H. Leblond and D. Mihalache, Phys. Rev. A 81, 063815 (2010); Rom. Rep. Phys. 63, 1254 (2011).

[19] M. B. Belonenko, E. V. Demushiva, and N. G. Lebedev, J. Russian Laser Res. 27, 457 (2006).

[20] M. B. Belonenko, N. G. Lebedev, and A. S. Popov, Pis'ma Zh. Eksp. Teor. Fiz. 91, 506 (2010) [JETP Lett. 91, 461 (2010)].

[21] R. Saito, M. Fujita, G. Dresselhaus, and M. S. Dresselhaus, Phys. Rev. B 46, 1804 (1992).

[22] M. A. Manna and V. Merle, Phys. Rev. E 57, 6206 (1998).

[23] H. Leblond and F. Sanchez, Phys. Rev. A 67, 013804 (2003). 